\title{
Introduction for the special issue on new paradigms in leukocyte trafficking, lessons for therapeutics
}

\author{
Francis W. Luscinskas • Beat A. Imhof
}

Received: 4 March 2014 / Accepted: 6 March 2014 / Published online: 9 April 2014

(C) Springer-Verlag Berlin Heidelberg 2014

This issue of Seminars in Immunology focuses on recent advances in how immune cells are recruited to sites of tissue and organ injury, viral or bacterial infections, or atherosclerotic lesions. This process of calling leukocytes to affected tissue is the hallmark of inflammation. As beneficial as these mechanisms are for host defense and survival, dysregulation can turn these protective processes against the body and induce chronic inflammatory pathologies such as rheumatoid arthritis, atherosclerosis, inflammatory bowel disease, or neuropathies. Understanding the molecular processes of immune cell homing will deliver more specific drugable targets for a modern therapeutic armamentarium to defeat inflammatory pathologies. The present issue assembles nine state-of-the-art reviews by outstanding experts in the field describing recent advances.

Leukocytes including granulocytes, monocytes, B lymphocytes, and $\mathrm{T}$ cell precursors are generated by the bone marrow, leave this organ via blood vessels, and are the origin of the body's immune defense system. Insight into the molecular mechanisms that lead to extravasation of leukocytes at sites of inflammation and injury has come from studies performed in vivo using intravital microscopy and from in vitro flow models that mimic physiological shear stress in postcapillary venules. The studies reported by many investigators have

This article is a contribution to the special issue on New paradigms in leukocyte trafficking, lessons for therapeutics - Guest Editors: F. W. Luscinskas and B. A. Imhof

F. W. Luscinskas $(\square)$

Center for Excellence in Vascular Biology, Department of Pathology,

Brigham and Women's Hospital and Harvard Medical School,

Boston, MA 02115, USA

e-mail: fluscinskas@rics.bwh.harvard.edu

B. A. Imhof

Department of Pathology and Immunology, University of Geneva,

CMU rue Michel Servet 1, 1211 Geneva, Switzerland

e-mail: Beat.Imhof@unige.ch resulted in the identification of the molecular mechanisms of leukocyte adhesion and resulted in a general multi-step model of rolling, triggering arrest and tight adhesion, and ultimately transmigration (Fig. 1).

Pathologists have already observed these phenomena using simple microscopes in the nineteenth century. One of them was Julius Cohnheim who defined margination and extravasation of white blood cells from inflamed veins and capillaries in a book that appeared in 1873 in Germany (Cohnheim, J.; Neue Untersuchungen über die Entzündung, Verlag von August Hirschwald, Berlin, Germany). Skipping forward to the early 2000 s, scientist gave the perception that molecules involved in so-called homing of leukocytes were mostly known and the processes were clearly described. However, this is by far not the case, and the exact mechanisms are more complex than previously imagined with many more molecules involved. Furthermore, processes that have yet to be elucidated include release of leukocytes from bone marrow, transendothelial migration of leukocytes, leukocyte migration within the tissue after leaving the blood vessel, and the migration of antigen-presenting cells to and within lymphatics.

In this issue, nine prominent investigators who have made major contributions in the field of immune cell homing kindly contributed comprehensive articles that provide up-to-date reviews of the literature and work from their laboratories. These articles provide new insight into the cellular and molecular mechanisms that regulate leukocyte adhesion to and transmigration across endothelium and epithelium into tissues, the molecular mechanisms that underlie the observed circadian rhythms dependent oscillations in leukocyte homing, the strategies leukocytes employ during interstitial migration in in vivo and in vitro models, and review concepts of lymphatic vessel structure and explore emerging experimental and clinical approaches that affect dendritic cell migration in lymphatics. 

Rolling
Selectins
Triggering
Chemokines
Transmigration
CAMs

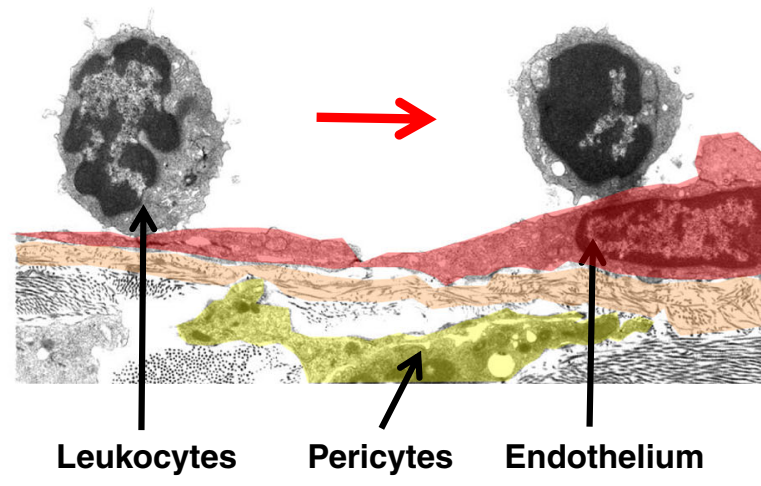

Fig. 1 Leukocytes homing: Leukocytes adhere to and traverse endothelium, basement membrane, pericytes and migrate into tissue. Leukocyte migration from the blood vessel into tissues mostly follows the same general mechanisms. The "Rolling" of leukocytes on luminal vascular endothelium is mediated by selectins that bind to carbohydrates on selectin ligands. The "Triggering" is mediated by chemokines and their signaling chemokine receptors expressed by the leukocytes. This signal leads to "Tight adhesion" by activating the leukocyte integrins LFA-1/ $\alpha \mathrm{L} \beta 2$ and $\alpha 4 \beta 1$ /VLA- 4 binding to the vascular Ig superfamily molecules ICAM or VCAM respectively. Specificity of leukocyte migration to

The complexities of monocyte lineages and differentiation is elegantly described by Swirski et al. [1], who explore how bone marrow stem cells can be mobilized, differentiate into monocytes and enter inflamed tissues. Several monocyte subclasses can emerge, in addition to the M1/M2 classification, and either stay in the blood to patrol the vascular endothelium or enter tissues and differentiate into macrophages or dendritic cells. These authors also point out that there is an embryoderived population that resides permanently in tissue and gives rise to macrophages that can proliferate in situ. Thus, not all monocyte/macrophages are controlled by the homing mechanisms during inflammation, and this should be kept in mind during design of therapeutic interventions.

Druzd et al. [2] describe the impact of circadian cycles on hematopoietic stem cell niches and immune cell homing, another important physiological process that has been ignored for a long time by immunologists. The story begins with the finding that symptoms of inflammatory diseases manifest during particular time periods of the day and that shift workers have higher incidences. Circadian rhythms and their master genes have been well described but their influence on molecular mechanisms controlling leukocyte migration and homing was discovered just recently. In their review, Druzd and colleagues [2] provide an authoritative overview of the molecular clock machinery and its interface with the hematopoietic stem cell niche and immune cell system. The authors highlight that the expression level of vascular adhesion molecules and chemokines and the number of migrating immune cells vary according to diurnal/nocturnal cycles, which can influence clinical outcomes of therapies.
The next three reviews provide a fresh overview of the molecular interaction of leukocytes with vascular endothelium and describe a whole series of novel types of adhesion molecules expressed by leukocytes or endothelium that also exert enzymatic activities either to reinforce adhesion or to prepare for the process of transendothelial migration. Transmigration through the endothelial monolayer and into the vessel wall is a complex process that only very recently was elucidated by sequential action of different vascular adhesion molecules. Furthermore, differential posttranslational modifications of endothelial cell-cell lateral junction proteins allow cells to regulate disparate but mechanistically similar inflammatory functions such as leukocyte transmigration and vascular permeability for solutes.

Salmi and Jalkanen [3] describe a novel series of cell surface molecules on leukocytes and vascular endothelial cells with adhesion and enzymatic activities. They include amine oxidases such as vascular adhesion protein 1 (VAP-1), the NAD metabolizing enzymes CD38 and CD157, and several peptidases, metalloproteinases, phosphorylases, or ectonucleotidases. These types of molecules constitute up to $4 \%$ of cell surface proteins, and their adhesive and enzymatic activity substantially influence the outcome of inflammatory diseases, mainly through an effect on leukocyte adhesion and migration. Function blocking antibodies as well as small inhibitory molecules against several of these dual function molecules are presently under clinical investigation. For VAP-1, they concern skin inflammations, rheumatoid arthritis, and ulcerative colitis. The review by Vestweber et al. [4] distills the essential cellular components, molecular 
mechanisms, and the complexities that underlie regulation of leukocyte transmigration and vascular permeability by the adherens junctions. The review highlights the critical role that vascular endothelial (VE)-cadherin (cadherin-5) plays in regulating leukocyte and solute passage through cell-cell lateral borders. Remarkably, Vestweber et al. [4] found that during inflammatory reactions, the phosphorylation state of two distinct tyrosine residues in the cytoplasmic tail of VE-cadherin acts as master controllers for either leukocyte diapedesis or vascular permeability [10]. The molecular mechanism employs specific tyrosine phosphatases and tyrosine kinase that modify the cytoplasmic tail of VE-cadherin. These data are the first to demonstrate that transendothelial migration and permeability are two differently controlled events. Sullivan and Muller [5] focus their effort on understanding transendothelial migration of leukocytes. They describe this homing step that follows rolling, triggering, and tight adhesion, itself as a multimolecular event in which adhesion molecules that include PECAM-1, CD99, or DNAM function sequentially during leukocyte migration across the endothelial layer. Notable is the so-called lateral border recycling compartment (LBRC) that operates as an intracellular membrane compartment which hosts a reservoir of these adhesion molecules that are mobilized and directed to sites of leukocyte transmigration.

Luissint et al. [6] provide a comprehensive review of the junctional adhesion molecule (JAM) family that is involved in controlling the function of tight junctions of vascular endothelium and mucosal epithelium in terms of leukocyte transmigration and permeability to solutes. This family of molecules also plays a role in angiogenesis, cell migration and proliferation. The distribution, expression profile, and signal transduction of the JAM family of proteins are dramatically modulated by inflammatory stimuli and thereby affect the integrity of the two types of junctional barriers. The role of recently described JAM family members Coxsackie and adenovirus receptor (CAR), endothelial cell-selective adhesion molecule (ESAM), junctional adhesion molecule-like protein (JAM-L) are discussed in the context of the leukocyte recruitment across vascular endothelial and mucosal epithelial barriers. The authors also remind readers that leukocyte transmigration for endothelial (apical to basal) is the opposite of transmigration of epithelial (basal to apical) monolayers. This important aspect is highlighted by the authors and has to be taken into account when therapeutic interventions are considered.

Once the leukocyte has breached the vasculature, it has to navigate within the interstitial space towards pathogens in an efficient spatiotemporal manner. Lämmermann and Germain [7] found and describe that this process is more complex than we previously thought. Much of our understanding of leukocyte migration at the molecular level has come from in vitro studies carried out in 2D systems using components of the extracellular matrix as paved roads. Leukocyte migration in 2D models relies on strong adhesive forces mediated by integrins. In contrast, recent studies examining leukocyte migration employing 3D network models with high-resolution intravital microscopy reveal that cell migration is less dependent on integrins and relies more on chemokines and highly ordered regulations of the leukocytes actin cytoskeleton, leading to amoeboid rather than adhesion molecule-mediated locomotion.

A novel concept of leukocyte interactions comes by a novel class of extracellular proteins that bridge the leukocyte to other cell types or to extracellular components. One of them is cysteine-rich molecule CCN1. Emre and Imhof [8] describe $\mathrm{CCN} 1$ as an extremely versatile compound that comprises ligands for a plethora cell adhesion integrins and matrix proteins such as laminin, fibronectin, and perlecan. Furthermore, this protein also captures growth factors such as BMPs, TGF $\beta$, or VEGF. It is easy to envision the concept that $\mathrm{CCN} 1$ produced by inflamed cells could influence adhesion and migration, formation of stem cell niches, or even influence cell death or survival, and this may include thymus development but also chronic inflammatory pathologies such bowel diseases.

Finally and often ignored is the molecular mechanism of recruitment of leukocytes to lymphatic vessels and drainage into lymphoid organs. Teijeira et al. [9] cover this aspect in depth for antigen-collecting and antigen-presenting dendritic cells (DCs). The authors highlight the concept that mainly chemokines are at the heart of DC migration towards the lymphoid capillaries but this depends on the type of DCs as there exist several subpopulations. Entry of DCs through the so-called flaps of the capillaries is dependent on adhesion and signaling molecules and guides DCs into the valve-containing collector vessel. The authors describe observations made by several laboratories using gene-manipulated mice. Molecules such as the integrin ligands ICAMs and VCAM, but also semaphorins seem to be involved. Thus, through manipulation of such mechanisms, fine-tuning of immune reactions may become possible in the near future.

In summary, recent years allowed significant advancement in our understanding in immune cell adhesion and homing. This will lead to novel therapeutic possibilities with improved selectivity and hopefully less side effects in treating inflammatory pathologies. The presented reviews pave the way for this eternal endeavor. The guest editors are indebted to the contributing authors for their time, expertise and energy in making possible this outstanding update of the field.

\section{References}

1. Swirski FK, Hilgendorf I, Robbins CS (2014) From proliferation to proliferation: monocyte lineage comes full circle. Semin Immunopathol. doi:10.1007/s00281-013-0409-1

2. Druzd D, de Juan A, Scheiermann C (2014) Circadian rhythms in leukocyte trafficking. Semin Immunopathol. doi:10.1007/s00281013-0414-4 
3. Salmi M, Jalkanen S (2014) Ectoenzymes in leukocyte migration and their therapeutic potential. Semin Immunopathol. doi:10.1007/ s00281-014-0417-9

4. Vestweber D, Wessel F, Nottebaum A (2014) Similarities and differences in the regulation of leukocyte extravasation and vascular permeability. Semin Immunopathol. doi:10.1007/ s00281-014-0419-7

5. Sullivan DP, Muller W (2013) Neutrophil and Monocyte Recruitment by PECAM, CD99, and Other Molecules via the LBRC. Semin Immunopathol. doi:10.1007/s00281-013-0412-6

6. Luissint A-C, Nusrat A, Parkos CA JAM related proteins in mucosal homeostasis and inflammation. Semin Immunopathol. doi:10.1007/ s00281-014-0421-0
7. Lämmermann T, Germain RN (2014) The multiple faces of leukocyte interstitial migration. Semin Immunopathol. doi:10.1007/s00281014-0418-8

8. Emre Y, Imhof B (2014) Matricellular protein CCN1/CYR61, a new player in inflammation and leukocyte trafficking. Semin Immunopathol. doi:10.1007/s00281-014-0420-1

9. Teijeira A, Russo E, Halin C DC homing to and within lymphatics. doi:10.1007/s00281-013-0410-8

10. Wessel F, Winderlich M, Holm M, Frye M, Rivera-Galdos R, Vockel M, Linnepe R, Ipe U, Stadtmann A, Zarbock A, Nottebaum AF, Vestweber D (2014) Leukocyte extravasation and vascular permeability are each controlled in vivo by different tyrosine residues of VE-cadherin. Nat Immunol 15:223-230 